\title{
Special issue on selected papers from NORCHIP 2008
}

\author{
Peeter Ellervee $\cdot$ Gert Jervan
}

Published online: 30 April 2010

(C) Springer Science+Business Media, LLC 2010

This special issue is composed of papers from the 26th NORCHIP conference that was held in Tallinn, Estonia on November 17-18, 2008. The NORCHIP conference is the main microelectronics event of the Nordic and Baltic countries. The technical program of NORCHIP 2008 contained 61 papers, divided into 16 regular sessions and two poster sessions and was attended by almost 100 participants.

Selected papers from the conference were invited for this special issue. The guest editors feel that the following seven papers that were chosen based on rigorous review all contain contributions that will be of interest to the Analog Integrated Circuits and Signal Processing readers.

In the first paper, Dan Sandström, Mikko Varonen, Mikko Kärkkäinen and Kari Halonen from Helsinki University of Technology, Finland, present a three-stage V-band amplifier implemented in 65-nm baseline CMOS technology. The matching circuitry and interconnects are implemented with slow-wave coplanar waveguides to study the feasibility of using this type of a transmission line in amplifier design. Measured power gain, noise figure and 1-dB output compression point at $60 \mathrm{GHz}$ are $13 \mathrm{~dB}$, $6.3 \mathrm{~dB}$ and $+4 \mathrm{dBm}$, respectively. The amplifier has $19.6 \mathrm{GHz}$ of $3 \mathrm{~dB}$ bandwidth, thus covering entirely the unlicensed band around $60 \mathrm{GHz}$.

The second paper is coming from Norwegian University of Science and Technology and University of Oulu, Finland. Simo Hietakangas, Jukka Typpö and Timo Rahkonen

\footnotetext{
P. Ellervee $(\bowtie) \cdot$ G. Jervan

Department of Computer Engineering, Tallinn University

of Technology, Tallinn, Estonia

e-mail: 1rv@cc.ttu.ee

G. Jervan

e-mail: gert.jervan@ati.ttu.ee
}

present a paper titled "Design of Integrated 1.6 GHz, $2 \mathrm{~W}$ Tuned RF Power Amplifier". This paper describes the design of an integrated tuned power amplifier specified to operate at Inmarsat satellite uplink frequencies from 1626.5-1660.5 MHz. The aim of the paper is to show experiences related to the design of switching high power radio frequency (RF) amplifiers (PA) with integrated output pulse-shaping. The amplifier was implemented onto a GaAs substrate with depletion mode high electron mobility transistors (FETs).

In the third paper Jonas Fritzin and Atila Alvandpour from Linköping University, Sweden describe the design and implementation of a power amplifier (PA) for 802.11n WLAN fabricated in $65 \mathrm{~nm}$ CMOS technology, including the circuit architecture, modeling and design of the transformers, the extraction of the layout parasitics, and the output matching network design, which is followed by the experimental results. The PA utilizes $3.3 \mathrm{~V}$ thick gate oxide $(5.2 \mathrm{~nm})$ transistors and a two-stage differential configuration with integrated transformers for input and interstage matching. The PA meets the EVM and spectral requirements for a $72.2 \mathrm{Mbit} / \mathrm{s}$, 64-QAM, 802.11n OFDM signal, at an average output power of $11.6 \mathrm{dBm}$, with an EVM of $3.8 \%$.

The fourth paper by Oliver Schmitz, Sven Karsten Hampel, Christian Orlob, Marc Tiebout and Ilona Rolfes from Universität Hannover, Germany is titled "Body Effect Up- and Down-Conversion Mixer Circuits for Low-Voltage Ultra-Wideband Operation". This paper presents fully differential up- and down-conversion mixer circuits manufactured in a triple well $45 \mathrm{~nm}$ CMOS process for low-voltage Ultra-Wideband transmitter and receiver applications. By presenting exemplary $45 \mathrm{~nm}$ CMOS bulk-driven mixer designs, the authors have pointed out flexible application of these designs within 
transmitter and receiver applications and the fundamental benefits of these mixer topologies for low voltage broadband operation.

The fifth paper is coming from Linköping University, Sweden. Timmy Sundström and Atila Alvandpour present a 6-bit 2.5-GS/s Flash ADC using Comparator Redundancy for Low Power in $90 \mathrm{~nm}$ CMOS. The aim of the paper is to show that redundancy can be used to achieve an overall energy efficient design at gigasample per second rate with the sole focus on designing for speed and low power at the building block level. As the mismatch induced comparator offsets will get worse with scaling, using comparator redundancy is a means for taking full advantage of decreased feature sizes in future CMOS processes, with higher speeds and lower power dissipation.

The authors of the sixth paper, Nashwa Abo Elneel, Erkan Aksoy, and Dietmar Schroeder from Hamburg University of Technology, Germany and CEA-LETI, Grenoble, France describe a novel adaptable analog/digital converter (ADC) that combines analog/digital conversion and entropy-coding for integrated data compression and low-power operation. The converter has high flexibility of operation in terms of adaptable resolution, conversion rate and input signal statistics. This feature allows to adaptively react to changes of the situation and to put the device in each case into the optimum configuration. Since the ADC can be configured on the fly to minimize power consumption, it is particularly suited for wireless sensor networks.

The seventh paper from University of Oulu by Ilkka Nissinen and Juha Kostamovaara presents noise and jitter characteristics of an on-chip voltage reference-locked ring oscillator used in the time-to-digital converter (TDC) of the integrated receiver of a pulsed time-of-flight laser rangefinder. The frequency of the ring oscillator, $683 \mathrm{MHz}$, was locked to the on-chip voltage reference by means of a frequency-to-voltage converter, resulting in better than $90 \mathrm{ppm} /{ }^{\circ} \mathrm{C}$ stability. The noise and jitter transfer characteristics of the loop were derived, and simulations were performed to see the effects of different noise types (white and $1 / \mathrm{f}$ noise) on the cumulative jitter of the locked ring oscillator.

These seven papers have undergone normal review. We would like to express our gratitude to the reviewers for the comments and suggestions that have improved quality of the papers.

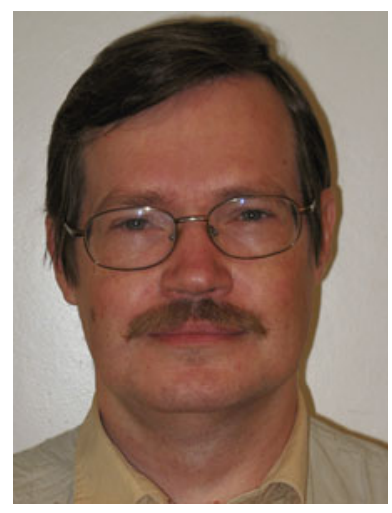

Peeter Ellervee is professor at the Department of Computer Engineering at Tallinn University of Technology, Estonia. He received his Dipl. Eng. degree from Tallinn University of Technology in 1984 and $\mathrm{PhD}$ degree from Royal Institute of Technology (KTH), Stockholm, Sweden in 2000. He in the editorial board of Elsevier's journal Microprocessors and Microsystems: Embedded Hardware Design (MICPRO). He has been a vice chair of Baltic Electronics Conferences 2008 \& 2010, and Norchip Conference 2008. He is a member of the management committee of Norchip Conferences. He belongs to the program committees of International Conference on Field Programmable Logic and Applications; European Workshop on Microelectronics Education; International Conference on Advances in Circuits, Electronics and Micro-electronics; Workshop on Reconfigurable Communication-centric Systems-on-Chip; and International Symposium on System-on-Chip. He has also served as a reviewer for several major conferences and journals, such as DAC, DATE, FPL, Euromicro DSD, MICPRO, JSA, JRC, IET CDT, IEEE TE, IEEE TVLSI. He has published more than 70 internationally reviewed papers and 3 book chapters in the fields of high- and logic level synthesis, and digital systems design.

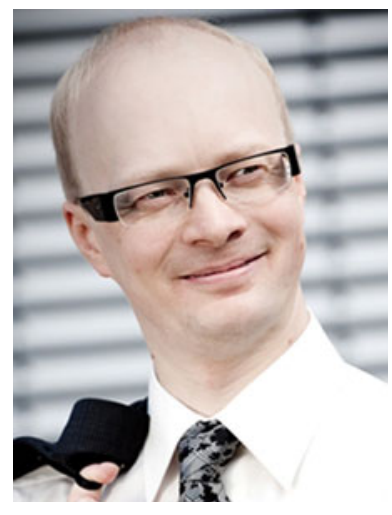

Gert Jervan is a senior research fellow at the Department of Computer Engineering at Tallinn University of Technology, Estonia. He received his MSc degree from Tallinn University of Technology in 1998 and Tech. Lic. and PhD degrees from Linköping University (LIU), Sweden in 2002 and 2005, respectively. He has been a special session chair of the 2010 Diagnostic Services in Network-on-Chips workshop (co-located with DAC 2010), vice program chair of Norchip 2008, general chair of the 19th EAEEEIE Annual Conference (2008) and was one of the organizers of the DATE 2008 Friday Workshop Impact of Process Variability on Design and Test. He belongs to the program committees of the Norchip Conference, International Conference on Architecture of Computing Systems and Workshop on Low Power Design Impact on Test and Reliability. He has also served as a reviewer for several conferences and journals, such as DATE, ITC, ETS, ATS, Euromicro DSD, MICPRO, IET CDT, IEEE Trans. on VLSI, IJERTCS, Integration, the VLSI Journal, JOLPE, IEEE Trans. on Education, IEEE Trans. on CAD, Journal of Parallel and Distributed Computing and others. He has published more than 50 internationally reviewed papers and 2 book chapters in the fields of test and diagnostics of digital systems, built-in self-test, reliability and fault tolerance. 